ApJS accepted

\title{
An Explicit Scheme for Incorporating Ambipolar Diffusion in a Magnetohydrodynamics Code
}

\author{
Eunwoo Choi ${ }^{1}$, Jongsoo Kim ${ }^{1,2}$, and Paul J. Wiita ${ }^{3,4}$
}

\begin{abstract}
We describe a method for incorporating ambipolar diffusion in the strong coupling approximation into a multidimensional magnetohydrodynamics code based on the total variation diminishing scheme. Contributions from ambipolar diffusion terms are included by explicit finite difference operators in a fully unsplit way, maintaining second order accuracy. The divergence-free condition of magnetic fields is exactly ensured at all times by a flux-interpolated constrained transport scheme. The super time stepping method is used to accelerate the timestep in high resolution calculations and/or in strong ambipolar diffusion. We perform two test problems, the steady-state oblique C-type shocks and the decay of Alfvén waves, confirming the accuracy and robustness of our numerical approach. Results from the simulations of the compressible MHD turbulence with ambipolar diffusion show the flexibility of our method as well as its ability to follow complex MHD flows in the presence of ambipolar diffusion. These simulations show that the dissipation rate of MHD turbulence is strongly affected by the strength of ambipolar diffusion.
\end{abstract}

Subject headings: diffusion — ISM: clouds — methods: numerical — MHD stars: formation - turbulence

\footnotetext{
${ }^{1}$ International Center for Astrophysics, Korea Astronomy and Space Science Institute, Daejeon 305-348, Korea; echoi@kasi.re.kr, jskim@kasi.re.kr.

${ }^{2}$ Cavendish Laboratory, University of Cambridge, JJ Thomson Avenue, Cambridge CB3 0HE, UK.

${ }^{3}$ Department of Physics and Astronomy, Georgia State University, P.O. Box 4106, Atlanta, GA 303024106; wiita@chara.gsu.edu.

${ }^{4}$ School of Natural Sciences, Institute for Advanced Study, Einstein Drive, Princeton, NJ 08540.
} 


\section{Introduction}

In galactic molecular clouds, ambipolar diffusion, which arises in partially ionized plasmas, is a key ingredient of the mechanism of star formation (e.g., Mestel \& Spitzer 1956; Mouschovias 1976; Shu et al. 1987). In the central portions of molecular clouds, the molecular gas is dense enough that recombination is nearly total, so that very low fractions $\left(\lesssim 10^{-7}\right)$ of the gas remains ionized while the rest of the gas is neutral (Myers 1985). This small residual ionization is usually attributed to cosmic rays, which can penetrate nearly all clouds. Ambipolar diffusion causes the relative drift of ions coupled to the magnetic field and neutrals in the molecular cloud cores and so it enable the cloud cores to collapse gravitationally.

Star formation assisted by ambipolar diffusion has been studied extensively in the context of magnetically subcritical or supercritical models (see e.g., Mouschovias \& Ciolek 1999; Desch \& Mouschovias 2001; Basu \& Ciolek 2004; Tassis \& Mouschovias 2007). In a current paradigm of star formation, magnetically supported molecular cloud cores must lose magnetic support through the action of ambipolar diffusion so that star formation can take place. Recent works have focused on the role of turbulence in the formation of protostellar cores (e.g., Nakamura \& Li 2008). Including the effect of turbulence on the mechanism of ambipolar diffusion can enhance the ambipolar diffusion rate (Fatuzzo \& Adams 2002; Zweibel 2002; Heitsch et al. 2004), so that the ambipolar diffusion timescale is significantly shorter than that estimated for a similar, but quiescent, medium. Using three-dimensional numerical simulations, Oishi \& Mac Low (2006) and Li et al. (2008) have studied the properties of turbulence with ambipolar diffusion in a two-fluid approximation, while Padoan et al. (2000) have investigated the heating through ambipolar diffusion in turbulent molecular clouds using a single-fluid approximation.

Shock waves in molecular clouds spread into steady-state continuous shocks, or C-type shocks, through ambipolar diffusion. If the shock speed is slower than the ion Alfvén speed but faster than the neutral sound speed, the ions coupled to magnetic fields drag the neutrals into the postshock region, producing a continuous structure (Draine 1980). These steady-state C-type shocks can, however, be unstable on a short enough timescale to be of astrophysical interest. Wardle (1991) showed that if the magnetic field lines are perturbed slightly and ions collect in the magnetic valleys, the ion-neutral friction may overcome the magnetic forces in the shock front and derive an exponentially growing instability.

Numerical treatments of ambipolar diffusion have been commonly derived from ideal magnetohydrodynamic (MHD) models. Extensive numerical methods including ambipolar diffusion have been proposed in the study of the dynamics of partially ionized plasmas within the frame of single or two fluid models (Tóth 1994; Mac Low et al. 1995; Mac Low \& Smith 1997; Smith \& Mac Low 1997; Stone 1997; Li et al. 2006; Tilley \& Balsara 2008). Mac Low et al. 
(1995) have described an explicit method for one-fluid ambipolar diffusion in the strong coupling limit, while Tóth (1994) has used a semi-implicit scheme for two-fluid ambipolar diffusion to investigate instability in C-type shocks. Tilley \& Balsara (2008) have also presented a semi-implicit method for ambipolar diffusion using a two-fluid approximation. Implicit schemes for the multifluid treatment of Hall diffusion and ambipolar diffusion have been suggested by Falle (2003) and O'Sullivan \& Downes (2006, 2007).

In this work we describe a fully explicit method for incorporating the single-fluid ambipolar diffusion into a multidimensional MHD code based on the total variation diminishing scheme. The divergence-free condition of the magnetic field is ensured by a flux-interpolated constrained transport scheme, and a super time stepping method is used in order to considerably accelerate the otherwise painfully short diffusion-driven time steps.

The organization of this paper is as follows. In $\S 2$ the MHD equations are presented along with the approximations we have made and in $\S 3$ our numerical methods are described in detail. Two test problems are presented in $\S 4$, while MHD turbulence simulations with significant ambipolar diffusion follow in $\S 5$. A summary is given in $\S 6$.

\section{MHD Equations with Ambipolar Diffusion}

We assume the strong coupling approximation, i.e., that the ion pressure and momentum are usually negligible in the weakly ionized plasma compared to those of the neutrals and so the magnetic force on the ions and the drag force exerted by the neutrals on the ions are almost equal. In this approximation the plasma can be represented as a single fluid. This single-fluid approximation turns out to be useful in the formation of molecular cloud cores through the process of ambipolar diffusion (Shu et al. 1987). To simplify the modeling of ambipolar diffusion here we assume isothermality with a constant sound speed in the ions and the neutrals and we ignore gravity.

The isothermal MHD equations including ambipolar diffusion can then be written as

$$
\begin{gathered}
\frac{\partial \rho}{\partial t}+\nabla \cdot(\rho \boldsymbol{v})=0, \\
\frac{\partial \boldsymbol{v}}{\partial t}+\boldsymbol{v} \cdot \boldsymbol{\nabla} \boldsymbol{v}+\frac{1}{\rho} \nabla p-\frac{1}{\rho}(\boldsymbol{\nabla} \times \boldsymbol{B}) \times \boldsymbol{B}=0, \\
\frac{\partial \boldsymbol{B}}{\partial t}-\nabla \times(\boldsymbol{v} \times \boldsymbol{B})=\nabla \times\left\{\left[\frac{1}{\gamma \rho_{i} \rho}(\boldsymbol{\nabla} \times \boldsymbol{B}) \times \boldsymbol{B}\right] \times \boldsymbol{B}\right\},
\end{gathered}
$$

with the additional constraint for the absence of magnetic monopoles,

$$
\boldsymbol{\nabla} \cdot \boldsymbol{B}=0 .
$$


Here the equation of state is $p=a^{2} \rho$, where $a$ is an isothermal sound speed, $\gamma$ is the collisional coupling constant between ions and neutrals, and $\rho_{i}$ is the ion density. The other variables $\rho, \boldsymbol{v}$, and $\boldsymbol{B}$ denote neutral density, neutral velocity, and magnetic field, respectively. We renormalize the magnetic field by defining $\boldsymbol{B} \equiv \boldsymbol{B} / \sqrt{4 \pi}$ throughout this paper so that the factor of $4 \pi$ does not appear in equations (2) and (3).

Although the ion density in molecular clouds depends on complicated physical balance between the cosmic-ray ionization of neutrals and the recombination of ions and electrons on charged grains, for the purpose of simplicity we assume that the ion density scales as a power of the neutral density (e.g., Elmegreen 1979)

$$
\rho_{i}=\rho_{i 0}\left(\frac{\rho}{\rho_{0}}\right)^{\alpha} .
$$

By setting $\alpha=0$ we further simplify by taking the ion density constant in this work, as these variations will not significantly affect the problems we treat. For conditions appropriate to molecular clouds, however, the choice of $\alpha \sim 0.5$ usually would be more realistic, with $\alpha \sim 0$ most applicable at very high densities where grains are the main charge carriers (e.g., Ciolek \& Mouschovias 1998). On the other hand, the ion velocity is obtained from the equation for the relative drift velocity between ions and neutrals,

$$
\boldsymbol{v}_{i}=\boldsymbol{v}+\frac{1}{\gamma \rho_{i} \rho}(\boldsymbol{\nabla} \times \boldsymbol{B}) \times \boldsymbol{B} .
$$

This equation shows that the drag force and the magnetic force on the ions are balanced and that the ion-neutral drift velocity $\boldsymbol{v}_{d}=\boldsymbol{v}_{i}-\boldsymbol{v}$ is always perpendicular to the magnetic field.

The basic effect of ambipolar diffusion on the magnetic field can be expressed in a diffusion coefficient $D$ (Shu et al. 1987) given by

$$
D=\tau c_{\mathrm{A}}^{2},
$$

where $\tau=1 / \gamma \rho_{i}$ is the mean collisional time between ions and neutrals and $c_{\mathrm{A}}=B / \sqrt{\rho}$ is the Alfvén speed. Then we can estimate the ambipolar diffusion timescale as

$$
t_{\mathrm{AD}}=\frac{L^{2}}{D}
$$

where $L$ is the characteristic length scale of magnetic field. 


\section{Numerical Methods}

\subsection{Source Term Integration}

The numerical scheme for solving the ideal MHD equations is described in previous works (Ryu et al. 1995, 1998; Kim et al. 1999). This method is based on the total variation diminishing (TVD) scheme (Harten 1983) which is an explicit Eulerian upwind scheme with a second-order accuracy in space and time. In the strong coupling approximation we can separate the ion density and velocity from the neutral density and velocity so that we can basically use the MHD TVD code to compute the evolution of the neutral density and velocity using mass and momentum conservation equations.

We now describe how to incorporate ambipolar diffusion terms into the induction equation. The induction equation can be rewritten in component form as

$$
\begin{aligned}
& \frac{\partial B_{x}}{\partial t}+\frac{\partial}{\partial y}\left(B_{x} v_{y}-B_{y} v_{x}\right)-\frac{\partial}{\partial z}\left(B_{z} v_{x}-B_{x} v_{z}\right)=\frac{\partial S_{z}}{\partial y}-\frac{\partial S_{y}}{\partial z} \\
& \frac{\partial B_{y}}{\partial t}+\frac{\partial}{\partial z}\left(B_{y} v_{z}-B_{z} v_{y}\right)-\frac{\partial}{\partial x}\left(B_{x} v_{y}-B_{y} v_{x}\right)=\frac{\partial S_{x}}{\partial z}-\frac{\partial S_{z}}{\partial x} \\
& \frac{\partial B_{z}}{\partial t}+\frac{\partial}{\partial x}\left(B_{z} v_{x}-B_{x} v_{z}\right)-\frac{\partial}{\partial y}\left(B_{y} v_{z}-B_{z} v_{y}\right)=\frac{\partial S_{y}}{\partial x}-\frac{\partial S_{x}}{\partial y} .
\end{aligned}
$$

Here the source term components are given by

$$
\begin{aligned}
& S_{x}=\frac{1}{\gamma \rho_{i} \rho}\left[\left(\frac{\partial B_{y}}{\partial x}-\frac{\partial B_{x}}{\partial y}\right) B_{x} B_{z}+\left(\frac{\partial B_{y}}{\partial z}-\frac{\partial B_{z}}{\partial y}\right)\left(B_{z}^{2}+B_{y}^{2}\right)+\left(\frac{\partial B_{x}}{\partial z}-\frac{\partial B_{z}}{\partial x}\right) B_{x} B_{y}\right], \\
& S_{y}=\frac{1}{\gamma \rho_{i} \rho}\left[\left(\frac{\partial B_{z}}{\partial y}-\frac{\partial B_{y}}{\partial z}\right) B_{y} B_{x}+\left(\frac{\partial B_{z}}{\partial x}-\frac{\partial B_{x}}{\partial z}\right)\left(B_{x}^{2}+B_{z}^{2}\right)+\left(\frac{\partial B_{y}}{\partial x}-\frac{\partial B_{x}}{\partial y}\right) B_{y} B_{z}\right], \\
& S_{z}=\frac{1}{\gamma \rho_{i} \rho}\left[\left(\frac{\partial B_{x}}{\partial z}-\frac{\partial B_{z}}{\partial x}\right) B_{z} B_{y}+\left(\frac{\partial B_{x}}{\partial y}-\frac{\partial B_{y}}{\partial x}\right)\left(B_{y}^{2}+B_{x}^{2}\right)+\left(\frac{\partial B_{z}}{\partial y}-\frac{\partial B_{y}}{\partial z}\right) B_{z} B_{x}\right] .
\end{aligned}
$$

Note that the source terms on the right-hand side of equations (9) to (11) have the same divergence form as the flux components on the left-hand side.

Standard second-order finite difference operators are applied to the explicit discretization of the source components. Here we define the source components at grid centers, $S_{x, i, j, k}$, $S_{y, i, j, k}$, and $S_{z, i, j, k}$, while the $n$-th components of the TVD flux vectors in each direction, $\bar{f}_{x, i+1 / 2, j, k}^{(n)}, \bar{f}_{y, i, j+1 / 2, k}^{(n)}$, and $\bar{f}_{z, i, j, k+1 / 2}^{(n)}$, are defined at face centers. The first four components of the TVD flux vectors, $\bar{f}^{(1)}$ through $\bar{f}^{(4)}$, are the upwind fluxes associated with the transport of mass and momentum, i.e., mass and momentum advection fluxes, and the last three 
components of the TVD flux vectors, $\bar{f}^{(5)}$ through $\bar{f}^{(7)}$, represent the components of the electric field. While keeping second-order accuracy, the source components can then be included in the TVD flux components as follows

$$
\begin{aligned}
& f_{x, i+1 / 2, j, k}^{(6)}=\bar{f}_{x, i+1 / 2, j, k}^{(6)}+\frac{1}{2}\left(S_{z, i, j, k}+S_{z, i+1, j, k}\right), \\
& f_{x, i+1 / 2, j, k}^{(7)}=\bar{f}_{x, i+1 / 2, j, k}^{(7)}-\frac{1}{2}\left(S_{y, i, j, k}+S_{y, i+1, j, k}\right), \\
& f_{y, i, j+1 / 2, k}^{(7)}=\bar{f}_{y, i, j+1 / 2, k}^{(7)}+\frac{1}{2}\left(S_{x, i, j, k}+S_{x, i, j+1, k}\right), \\
& f_{y, i, j+1 / 2, k}^{(5)}=\bar{f}_{y, i, j+1 / 2, k}^{(5)}-\frac{1}{2}\left(S_{z, i, j, k}+S_{z, i, j+1, k}\right), \\
& f_{z, i, j, k+1 / 2}^{(5)}=\bar{f}_{z, i, j, k+1 / 2}^{(5)}+\frac{1}{2}\left(S_{y, i, j, k}+S_{y, i, j, k+1}\right), \\
& f_{z, i, j, k+1 / 2}^{(6)}=\bar{f}_{z, i, j, k+1 / 2}^{(6)}-\frac{1}{2}\left(S_{x, i, j, k}+S_{x, i, j, k+1}\right) .
\end{aligned}
$$

Since the TVD scheme has second-order accuracy, the above second-order interpolation of the source components should be adequate. The contributions of the source components are added in a fully unsplit way after all the TVD flux components are updated through the TVD step. These total advective fluxes at face centers, $f_{x, i+1 / 2, j, k}^{(n)}, f_{y, i, j+1 / 2, k}^{(n)}$, and $f_{z, i, j, k+1 / 2}^{(n)}$, are used to enforce $\boldsymbol{\nabla} \cdot \boldsymbol{B}=0$ as described in the following subsection as well as to update the magnetic field components to the next time step.

\subsection{Divergence-Free Condition}

Analytically the divergence-free condition $\boldsymbol{\nabla} \cdot \boldsymbol{B}=0$ is maintained if the condition holds for the initial magnetic field, but numerically the divergence of the magnetic field will not be exactly zero due to numerical discretization and dimensional splitting. Several schemes to maintain $\boldsymbol{\nabla} \cdot \boldsymbol{B}=0$ constraint have been suggested and used in numerical MHD (see Tóth 2000). Evans \& Hawley (1988) suggested the constrained transport (CT) scheme which used a specific finite difference discretization on a staggered mesh to satisfy the divergencefree constraint. The flux-interpolated CT schemes (Ryu et al. 1998; Balsara \& Spicer 1999) have introduced a new staggered magnetic field variable which is updated by simple finite differences using the interpolated fluxes. We have found the flux-interpolated CT scheme to be effective for incorporating ambipolar diffusion and have followed the approach suggested by Balsara \& Spicer (1999). 
Using the total fluxes at face centers, equations (15) to (20), the advective fluxes at grid edges are reconstructed with second-order accuracy as follows

$$
\begin{aligned}
& \Omega_{x, i, j+1 / 2, k+1 / 2}=\frac{1}{4}\left(f_{z, i, j, k+1 / 2}^{(6)}+f_{z, i, j+1, k+1 / 2}^{(6)}-f_{y, i, j+1 / 2, k}^{(7)}-f_{y, i, j+1 / 2, k+1}^{(7)}\right), \\
& \Omega_{y, i+1 / 2, j, k+1 / 2}=\frac{1}{4}\left(f_{x, i+1 / 2, j, k}^{(7)}+f_{x, i+1 / 2, j, k+1}^{(7)}-f_{z, i, j, k+1 / 2}^{(5)}-f_{z, i+1, j, k+1 / 2}^{(5)}\right), \\
& \Omega_{z, i+1 / 2, j+1 / 2, k}=\frac{1}{4}\left(f_{y, i, j+1 / 2, k}^{(5)}+f_{y, i+1, j+1 / 2, k}^{(5)}-f_{x, i+1 / 2, j, k}^{(6)}-f_{x, i+1 / 2, j+1, k}^{(6)}\right) .
\end{aligned}
$$

Then the magnetic field components at face centers are updated as

$$
\begin{aligned}
b_{x, i+1 / 2, j, k}^{n+1}=b_{x, i+1 / 2, j, k}^{n} & -\frac{\Delta t}{\Delta y}\left(\Omega_{z, i+1 / 2, j+1 / 2, k}-\Omega_{z, i+1 / 2, j-1 / 2, k}\right) \\
& +\frac{\Delta t}{\Delta z}\left(\Omega_{y, i+1 / 2, j, k+1 / 2}-\Omega_{y, i+1 / 2, j, k-1 / 2}\right) \\
b_{y, i, j+1 / 2, k}^{n+1}=b_{y, i, j+1 / 2, k}^{n} & -\frac{\Delta t}{\Delta z}\left(\Omega_{x, i, j+1 / 2, k+1 / 2}-\Omega_{x, i, j+1 / 2, k-1 / 2}\right) \\
& +\frac{\Delta t}{\Delta x}\left(\Omega_{z, i+1 / 2, j+1 / 2, k}-\Omega_{z, i-1 / 2, j+1 / 2, k}\right) \\
b_{z, i, j, k+1 / 2}^{n+1}=b_{z, i, j, k+1 / 2}^{n} & -\frac{\Delta t}{\Delta x}\left(\Omega_{y, i+1 / 2, j, k+1 / 2}-\Omega_{y, i-1 / 2, j, k+1 / 2}\right) \\
& +\frac{\Delta t}{\Delta y}\left(\Omega_{x, i, j+1 / 2, k+1 / 2}-\Omega_{x, i, j-1 / 2, k+1 / 2}\right) .
\end{aligned}
$$

It is straightforward to show that $\boldsymbol{\nabla} \cdot \boldsymbol{b}^{n+1}=\boldsymbol{\nabla} \cdot \boldsymbol{b}^{n}=0$ if the numerical divergence of $\boldsymbol{b}$ is initially zero.

In the TVD scheme for MHD, all fluid quantities are defined at grid centers. Thus the magnetic field components at grid centers are interpolated as

$$
\begin{aligned}
B_{x, i, j, k} & =\frac{1}{2}\left(b_{x, i+1 / 2, j, k}+b_{x, i-1 / 2, j, k}\right), \\
B_{y, i, j, k} & =\frac{1}{2}\left(b_{y, i, j+1 / 2, k}+b_{y, i, j-1 / 2, k}\right), \\
B_{z, i, j, k} & =\frac{1}{2}\left(b_{z, i, j, k+1 / 2}+b_{z, i, j, k-1 / 2}\right) .
\end{aligned}
$$

Note that the above arithmetic interpolation will be sufficient to maintain second-order accuracy. 


\subsection{Super Time Stepping}

The time step for ambipolar diffusion is proportional to the square of the grid size in the single fluid approximation, so the explicit treatment of ambipolar diffusion terms leads to very small time steps (Mac Low et al. 1995). In the two fluid approximation that treats ions and neutrals separately, including the ion momentum equation severely limits the time steps via a very restrictive stability criterion. To resolve this problem, previous papers have proposed different solutions. For instance, Li et al. (2006) proposed a "heavy-ion" approximation to speed up the time steps, while Nakamura \& Li (2008) set a density threshold below which the ambipolar diffusion rate is set to zero to avoid this problem. In this work we adopt the "super time stepping" approach (Alexiades et al. 1996) to increase the effective time interval and allow much faster computations for ambipolar diffusion. O'Sullivan \& Downes (2006, 2007) also used this strategy in their multifluid MHD models.

The super time stepping technique considerably accelerates the explicit schemes for parabolic problems (Alexiades et al. 1996). The key advantage of this approach is that it demands stability over large compound time steps, rather than over each of the constituent substeps. In this method, the state vector is evolved over a super time step,

$$
\Delta t_{\mathrm{STS}}=\Delta t_{\mathrm{AD}} \frac{n}{2 \sqrt{\nu}}\left[\frac{(1+\sqrt{\nu})^{2 n}-(1-\sqrt{\nu})^{2 n}}{(1+\sqrt{\nu})^{2 n}+(1-\sqrt{\nu})^{2 n}}\right],
$$

where $\Delta t_{\mathrm{AD}}$ is the nominal ambipolar diffusion timestep, $n$ is the number of substeps, and

$\nu$ is a fuzzy factor $(0<\nu<1)$. This super time step is defined as $\Delta t_{\mathrm{STS}}=\sum_{j=1}^{n} \Delta \tau_{j}$, and consists of the substeps, $\Delta \tau_{j}$, which are given by

$$
\Delta \tau_{j}=\Delta t_{\mathrm{AD}}\left[(\nu-1) \cos \left(\frac{2 j-1}{n} \frac{\pi}{2}\right)+\nu+1\right]^{-1} .
$$

It has been proven that $\Delta t_{\mathrm{STS}} \rightarrow n^{2} \Delta t_{\mathrm{AD}}$ as $\nu \rightarrow 0$ so that the super time step approach is asymptotically $n$ times faster than the standard explicit scheme (Alexiades et al. 1996). However, the $\nu$ parameter must be properly chosen for each problem in order to achieve optimality and stability of performance. For diffusion-dominated problems, $\Delta t_{\mathrm{AD}}$ drops below the typical Courant time step, $\Delta t_{\text {Cour }}$ (i.e., $\Delta t_{\mathrm{AD}}<\Delta t_{\mathrm{STS}} \leq \Delta t_{\text {Cour }}$ ). Thus, if $\Delta t_{\mathrm{STS}}$ is taken to be the Courant time step, the super time stepping method requires roughly $\sqrt{\Delta t_{\text {Cour }} / \Delta t_{\mathrm{AD}}}$ substeps.

In addition to allowing larger effective time steps, the super time stepping approach offers relatively simple implementation since it is a first order method. We have successfully applied this approach to the linear and nonlinear ambipolar diffusion problems presented in the following sections. Our numerical results confirm the efficiency and accuracy of the super time stepping approach, as previously implemented in other approximations. 


\section{Test Problems}

\subsection{Oblique C-type Shocks}

We compute the structure of oblique C-type shocks in order to test the numerical methods described in the previous section. The steady state structure of a C-type shock is characterized conveniently by the shock length scale defined as

$$
L_{s}=\tau c_{\mathrm{A}}
$$

Following Mac Low et al. (1995), the steady state equations are solved by setting $\partial_{t}=\partial_{y}=$ $\partial_{z}=0$ in equations (1) to (3), which then can be reduced to a single ordinary differential equation for $\rho$. The steady state solution can be obtained through the numerical integration of the ordinary differential equation. In a separate code we numerically integrate the ordinary differential equation using the fourth-order Runge-Kutta method. This solution is specified by the three parameters, the sonic Mach number $M$, the Alfvén Mach number $M_{\mathrm{A}}$, and the angle $\theta$ between the shock normal and the magnetic field.

To generate C-type shocks we set up a two-dimensional shock heating problem. Initially a gas with a neutral density $\rho$ propagates with a velocity $\boldsymbol{v}$ against a reflecting wall placed at $x=0$ in the uniform magnetic field $\boldsymbol{B}$ that lies at an angle $\theta$ to the $x$-axis. As the gas hits the reflecting wall, both the fluid and the magnetic field are compressed, the gas is heated and a reverse shock is produced. The ion-neutral friction drags the neutral gas into the postshock region, and finally the steady-state C-type shock is built up, yielding the appropriate continuous transition. The parameters we chose for this problem are $a=0.1$, $\gamma=1, \rho_{i}=10^{-5}, \rho=1$, and $\boldsymbol{B}=B_{0 x} \hat{\boldsymbol{x}}+B_{0 y} \hat{\boldsymbol{y}}$ with $B_{0 x}=B_{0 y}=1 / \sqrt{2}$. This problem has been set up with two inflow velocities, $\boldsymbol{v}=-4.45 \hat{\boldsymbol{x}}$ and $-9.47 \hat{\boldsymbol{x}}$, which correspond to the shock velocities $v_{s}=5$ and 10 for our chosen parameters. This gives $M=v_{s} / a=50$ and $100, M_{\mathrm{A}}=v_{s} / c_{\mathrm{A}}=5$ and 10 , and $\theta=\tan ^{-1}\left(B_{0 y} / B_{0 x}\right)=\pi / 4$. The computations have been done in a two-dimensional box of $x=y=[0, L]$ with $L=20 L_{s}$ using $128^{2}$ cells. Outflow boundary conditions are used except for a reflecting boundary imposed at $x=0$.

The parameters for the run shown in Figure1(a) are $M=50, M_{\mathrm{A}}=5$, and $\theta=\pi / 4$ and those for the run shown in Figure 1(b) are $M=100, M_{\mathrm{A}}=10, \theta=\pi / 4$. In Figure 1, the structure of neutral density, neutral (red) and ion (blue) velocity components, and magnetic field components from numerical calculations are marked with open circles and compared to analytic solutions plotted with solid lines. Structures are measured along the $x$-direction before the shock reaches the outer boundary. The spike in the neutral density seen in a few cells near the reflecting wall is the overheating phenomenon. This is purely a numerical artifact that most finite difference schemes applied to the shock heating problem intrinsically 
possess since they cannot compute the jump condition across strong shocks within a single cell. In all the flow variables, the structure of the C-type shock clearly forms. Figure 1 shows the excellent agreement between the numerical solutions and the analytic solutions for the steady-state C-type shocks, demonstrating the accuracy of our numerical methods.

The accuracy of numerical solutions depends on the number of cells spanned by the box size $L$. So we have run the case of the test in Figure 1(a) with different numerical resolutions to check the convergence properties. Except for the resolutions the initial conditions are identical to those used in the test in Figure 1(a). We have computed the mean errors for neutral density defined by $\bar{E}(\rho)=\sum_{i, j}\left|\rho_{i, j}^{n}-\rho_{i, j}^{a}\right| / \sum_{i, j}\left|\rho_{i, j}^{a}\right|$, where the superscript $n$ represents numerical solution and the superscript $a$ represents analytic solution. The resolutions of $16^{2}, 32^{2}, 64^{2}, 128^{2}$, and $256^{2}$ cells give the mean errors of $1.33,1.29,1.28$, 1.27, and 1.27, respectively. As expected, the mean errors for neutral density converge as the numerical resolution increases. In this convergence test we also see that there are clear trends toward convergence in mean errors for velocity and magnetic field, and that a $128^{2}$ grid is sufficient to treat this problem.

\subsection{Decay of Alfvén Waves}

The propagation of Alfvén waves in a weakly ionized plasma provides an effective tool for testing the dynamics of ambipolar diffusion. Kulsrud \& Pearce (1969) first showed that ambipolar diffusion can prevent the propagation of Alfvén waves in a partially ionized medium. In the strong coupling approximation Balsara (1996) gives an explicit quadratic dispersion relation for Alfvén waves,

$$
\omega^{2}+i \frac{c_{\mathrm{A}}^{2} k^{2}}{\gamma \rho_{i}} \omega-c_{\mathrm{A}}^{2} k^{2}=0,
$$

where $\omega=\omega_{R}+i \omega_{I}$ is the complex angular frequency of the wave and $k$ is a real wavenumber. It is clear in the above equation that the Alfvén waves always propagate when $k<2 \gamma \rho_{i} / c_{\mathrm{A}}$ (i.e., $\omega_{R} \neq 0$ ). In order to test the propagation of Alfvén waves in the strong coupling limit, we have followed the evolution of a standing wave in numerical calculations and compared the oscillation frequency and decay rate of the wave to the analytic results. Damped

oscillations of standing waves have been long studied (e.g., Morse \& Ingard 1986) and the time-dependence of the first normal mode is described by

$$
h(t)=h_{0}\left|\sin \left(\omega_{R} t\right)\right| e^{\omega_{I} t}
$$

where $h_{0}$ is the initial amplitude of the wave.

In our test of the decay of Alfvén waves, we have used a standing wave formed along 
the diagonal on $x-y$ plane with initial velocity

$$
\boldsymbol{v}=v_{\mathrm{amp}} c_{\mathrm{A}} \sin \left(k_{x} x+k_{y} y\right) \hat{\boldsymbol{z}}
$$

The background density and magnetic field have been set to be uniform with $\rho=1$ and $\boldsymbol{B}=B_{0} \hat{\boldsymbol{x}}$ with $B_{0}=1$. This gives the characteristic Alfvén speed $c_{\mathrm{A}}=B / \sqrt{2 \rho}=0.7071$. Here the initial peak amplitude has been set to $v_{\mathrm{amp}}=0.1$ and the wavenumbers have been set to $k_{x}=k_{y}=2 \pi / L$ so the total wavenumber is $k=\sqrt{k_{x}^{2}+k_{y}^{2}}$. We choose collisional coupling constants, $\gamma=100,500$, and 1000 with $a=1$ and $\rho_{i}=0.1$ in this test. The calculations have been done in a computational box of $x=y=z=[0, L]$ with $L=1$ using $128^{3}$ cells. Boundary conditions are periodic in the $x$ - and $y$-directions and outflow in the $z$-direction.

Figure 2 shows the time evolution of the (spatially) root mean square magnetic field in the $z$-direction, $<\delta B_{z}^{2}>^{1 / 2}$, for three different collisional coupling constants $\gamma=1000$ (top), 500 (middle), and 100 (bottom) in the test of the decay of Alfvén waves. Our numerical results are marked with open circles while the theoretical predictions from equation (34) are plotted as solid lines. The oscillation frequencies and decay rates from these numerical calculations fit very well to those from the theoretical predictions, confirming that our numerical methods are accurate. Based on the time evolution of the standing wave in Figure 2, the propagation of Alfvén waves is significantly suppressed with decreasing collisional coupling constant $\gamma$.

By fitting the time evolution of $\left\langle\delta B_{z}^{2}>^{1 / 2}\right.$ to theoretical curves from equation (34) we find the numerical data for the complex angular frequency $\omega$, whose real and imaginary parts correspond to the oscillation frequency and decay rate of the standing wave, respectively. We repeated the calculation of Figure2 for $\gamma=100$, for eight wavenumbers ranging from $2 \pi / 16$ to

$6 \sqrt{2} \pi$, collecting the data for the oscillation frequencies $\omega_{R}$ and the decay rates $\omega_{I}$. In Figure 3 we show those "experimental" results together with the analytic solution of equation (33). Oscillation frequencies (red) and decay rates (blue) found from the numerical experiments are represented with filled circles and the analytic solutions are drawn as solid lines. In Figure 3 the very good agreement between numerical data and theoretical predictions for different wavenumbers shows the accuracy and flexibility of our numerical methods.

\section{MHD Turbulence Simulations}

In this section we present, as a first practical problem using this code, simulations of the compressible MHD turbulence in the presence of ambipolar diffusion. A simulation of turbulent ambipolar diffusion was studied by Padoan et al. (2000) in the strong coupling 
approximation and by Oishi \& Mac Low (2006) and Li et al. (2008) in the two-fluid approximation. We have performed this simulation to confirm the validation of our numerical methods for including ambipolar diffusion as well as to investigate the role of ambipolar diffusion in the dissipation of compressible MHD turbulence.

To characterize the MHD simulations, we define the strength of the magnetic field in terms of the parameter

$$
\beta \equiv \frac{a^{2}}{c_{\mathrm{A}}^{2}} .
$$

Note that our definition of $\beta$ differs by a factor of 2 from the usual plasma $\beta$, the ratio of gas pressure to magnetic pressure. For MHD turbulent flows with the root mean square velocity $v_{\text {rms }}$, the importance of magnetic fields on the dynamics of gas is characterized by the Alfvén Mach number $M_{\mathrm{A}}=v_{\mathrm{rms}} / c_{\mathrm{A}}=\sqrt{\beta} M$, where the sonic Mach number $M$ is given by $M=v_{\mathrm{rms}} / a$. The effect of ambipolar diffusion on Alfvénic turbulence of scale $L$ is measured through the ambipolar diffusion Reynolds number (Balsara 1996) defined by

$$
R_{\mathrm{AD}} \equiv \frac{v_{\mathrm{rms}} L}{\tau c_{\mathrm{A}}^{2}}
$$

A sufficiently large ambipolar diffusion Reynolds number implies that the importance of ambipolar diffusion to the turbulent flow on the scale $L$ becomes vanishingly small. The ambipolar diffusion length scale $l_{\mathrm{AD}}$ is then defined as a characteristic length scale at which the ambipolar diffusion Reynolds number becomes unity, i.e., $l_{\mathrm{AD}}=\tau c_{\mathrm{A}}^{2} / v_{\mathrm{rms}}$.

We consider two types of MHD turbulence models driven according to the method described in Stone et al. (1998). One is turbulence decaying from saturated initial velocity perturbations, and the other is forced turbulence in which velocity perturbations are added at constant time intervals. In both decaying and forced turbulence, the velocity perturbations $\delta v$ are generated from a Gaussian random field with a power spectrum

$$
P(k) \equiv \delta v^{2}(k) \propto k^{6} \exp \left(-8 k / k_{p}\right)
$$

where the power spectrum peaks at $k_{p}=4(2 \pi / L)$. The velocity perturbations are subject to the constraints that $\boldsymbol{\nabla} \cdot \delta \boldsymbol{v}=0$ and no net momentum is added by the velocity perturbations, $<\rho \delta \boldsymbol{v}>=0$. The perturbations are normalized so that the initial kinetic energy $\delta E_{K}=50$ for decaying turbulence and a constant kinetic energy input rate $\dot{E}_{K}=500$ is injected at regular time intervals $\delta t=0.001$ for forced turbulence.

The simulation parameters for both decaying models and forced models are summarized in Table1. According to the values of $\gamma$ varying from $\infty$ to 100, we denote decaying models as D1 to D3 and forced models as F1 to F3. In Table 1, the flow time is defined as $t_{f}=L / v_{\mathrm{rms}}$, 
and $t_{\text {end }}$ is the simulation end time. The model simulations have been set up with uniform neutral density $\rho=1$ and uniform magnetic field $\boldsymbol{B}=B_{0} \hat{\boldsymbol{x}}$ with $B_{0}=1$. The isothermal sound speed and ion density are assumed to be constant with $a=1$ and $\rho_{i}=0.01$. The simulations have been done in a periodic box of $x=y=z=[0, L]$ with $L=1$ using $128^{3}$ cells.

In Figures 4(a) and (b) we present images of the logarithms of the neutral density for models D1 (top) and D3 (bottom) at $t=1 t_{f}$ and for models F1 (top) and F3 (bottom) at $t=1 t_{f}$, respectively. The images are slices through the $x-y$ plane at $z=0.5$. The overall density features for both decaying turbulence and forced turbulence models are roughly the same, even though the turbulence driving pattern is different. Small-scale knots and filaments are produced in the absence of ambipolar diffusion (models D1 and F1). By introducing strong ambipolar diffusion (i.e., reducing the collisional coupling constant to 10), the density structures diffuse out, resulting in larger, smoother density structures, as shown in models D3 and F3. The results for models D2 and F2 are intermediate and are not shown. We note that the ranges of observed densities are smaller in the cases where ambipolar diffusion is strong, regardless of whether the turbulence is decaying or forced.

Figure 5 shows the time evolution of the total energy, defined as the sum of the kinetic energy and the energy of the perturbed magnetic field, $E_{\text {tot }}=E_{K}+E_{B}$, where

$$
E_{K}=\frac{1}{2} \int_{V} \rho v^{2} d V
$$

and

$$
E_{B}=\frac{1}{2} \int_{V}\left(B^{2}-B_{0}^{2}\right) d V
$$

with $d V=d x d y d z$. The evolution of the total energy for the decaying models, D1 (black), D2 (blue), and D3 (red) are plotted in Figure 5(a). After initial plateau phases all these models lose their initial energies rapidly, decaying nearly as power-laws with time (with indices between 1.1 to 1.3). The rate of the turbulent energy decay significantly increases with decreases in the collisional coupling constant, and thus we see that the turbulent decay rate can be strongly affected by differences in ambipolar diffusion. The somewhat more rapid decline of the total energy found for model D1 than for a similar simulation by Stone et al. (1998) can be understood in terms of the slightly lower resolution and higher initial energy in our simulation. In Figure 5(b), the evolution of the total energy for the forced models, F1 (black), F2 (blue), and F3 (red) are shown. In all the forced models the total energy rises steeply and then saturates at final states since the dissipation rate balances the input power. The amplitude of the final saturated energy level decreases with decreases in the collisional coupling constant, again showing that the dissipation rate increases as the strength of ambipolar diffusion increases. 


\section{Summary}

In this paper we describe specific numerical methods for incorporating ambipolar diffusion into a multidimensional MHD code based on the total variation diminishing scheme. We assume the strong coupling approximation, that magnetic force and the neutral-ion drag force in weakly ionized plasmas are almost equal and so the plasma can be treated as a single fluid. Since our numerical methods described in this paper are fully explicit and maintain a second-order accuracy, it is straightforward to extend them to parallelized versions and other geometries. The divergence-free constraint on the magnetic field has been exactly enforced through the flux-interpolated constraint transport scheme at all times. By using the super time stepping method to accelerate the timestep for ambipolar diffusion, we remove the severe restriction on the stable timestep that would arise at high numerical resolution and/or in strong ambipolar diffusion.

Ambipolar diffusion has been tested through the direct comparison with analytic solutions of diffusion problems. We have computed test problems that include oblique C-type shocks and the decay of Alfvén waves. For both of these test problems, comparisons of numerical results to analytic solutions are possible and they demonstrate the good accuracy and robustness of our methods. We have also performed simulations of the compressible MHD turbulence in the presence of ambipolar diffusion and they confirm the ability of our code to follow complex MHD flows. We have shown that the dissipation rate of MHD turbulence is strongly affected by the strength of ambipolar diffusion in both decaying turbulence and forced turbulence.

This multidimensional MHD code incorporating an explicit scheme for solving the ambipolar diffusion term allows us to study astrophysical systems such as molecular cloud cores and protostellar discs in which ambipolar diffusion is thought to be important. Currently this code is being used to study the evolution of compressible MHD turbulence with ambipolar diffusion, and results of three-dimensional, high resolution MHD simulations will be reported elsewhere.

This work utilized a high performance cluster at the Korea Astronomy and Space Science Institute (KASI). EC was supported by the KASI Postdoctoral Fellowship. JK was supported by the Korea Science and Engineering Foundation through the Astrophysical Research Center for the Structure and Evolution of Cosmos and by the Korea Foundation for International Cooperation of Science and Technology through grant K20702020016-07E0200-01610. 


\section{REFERENCES}

Alexiades, V., Amiez, G., \& Gremaud, P.-A. 1996, Comm. Num. Meth. Eng., 12, 31

Balsara, D. S. 1996, ApJ, 465, 775

Balsara, D. S., \& Spicer, D. S. 1999, J. Comput. Phys., 149, 270

Basu, S., \& Ciolek, G. E. 2004, ApJ, 607, L39

Ciolek, G. E., \& Mouschovias, T. Ch. 1998, ApJ, 504, 280

Desch, S. J., \& Mouschovias, T. Ch. 2001, ApJ, 550, 314

Draine, B. T. 1980, ApJ, 241, 1021

Elmegreen, B. G. 1979, ApJ, 232, 729

Evans, C. R., \& Hawley, J. F. 1988, ApJ, 332, 659

Falle, S. A. E. G. 2003, MNRAS, 344, 1210

Fatuzzo, M., \& Adams, F. C. 2002, ApJ, 570, 210

Harten, A. 1983, J. Comput. Phys., 49, 357

Heitsch, F., Zweibel, E. G., Slyz, A. D., \& Devriendt, J. E. G. 2004, ApJ, 603, 165

Kim, J., Ryu, D., Jones, T. W., \& Hong, S. S. 1999, ApJ, 514, 506

Kulsrud, R., \& Pearce, W. P. 1969, ApJ, 156, 445

Li, P. S., McKee, C. F., \& Klein, R. I. 2006, ApJ, 653, 1280

Li, P. S., McKee, C. F., Klein, R. I., \& Fisher, R. T. 2008, ApJ, 684, 380

Mac Low, M.-M., Norman, M. L., Königl, A., \& Wardle, M. 1995, ApJ, 442, 726

Mac Low, M.-M., \& Smith, M. D. 1997, ApJ, 491, 596

Mestel, L., \& Spitzer, L., Jr. 1956, MNRAS, 116, 503

Morse, P. M., \& Ingard, K. U. 1986, Theoretical Acoustics (Princeton: Princeton Univ. Press)

Mouschovias, T. Ch. 1976, ApJ, 207, 141 
Mouschovias, T. Ch., \& Ciolek, G. E. 1999, in The Origin of Stars and Planetary Systems, ed. C. J. Lada \& N. D. Kylafis (Dordrecht: Kluwer), 305

Myers, P. C. 1985, in Protostars and Planets II, ed. D. C. Black \& M. S. Matthews (Tucson: Univ. Arizona Press), 81

Nakamura, F., \& Li, Z.-Y. 2008, ApJ, 687, 354

Oishi, J. S., \& Mac Low, M.-M. 2006, ApJ, 638, 281

O’Sullivan, S., \& Downes, T. P. 2006, MNRAS, 366, 1329

O’Sullivan, S., \& Downes, T. P. 2007, MNRAS, 376, 1648

Padoan, P., Zweibel, E., \& Nordlund, A. 2000, ApJ, 540, 332

Ryu, D., Jones, T. W., \& Frank, A. 1995, ApJ, 452, 785

Ryu, D., Miniati, F., Jones, T. W., \& Frank, A. 1998, ApJ, 509, 244

Shu, F. H., Adams, F. C., \& Lizano, S. 1987, ARA\&A, 25, 23

Smith, M. D., \& Mac Low, M.-M. 1997, A\&A, 326, 801

Stone, J. M. 1997, ApJ, 487, 271

Stone, J. M., Ostriker, E. C., \& Gammie, C. F. 1998, ApJ, 508, L99

Tassis, K., \& Mouschovias, T. Ch. 2007, ApJ, 660, 370

Tilley, D. A., \& Balsara, D. S. 2008, MNRAS, 389, 1058

Tóth, G. 1994, ApJ, 425, 171

Tóth, G. 2000, J. Comput. Phys., 161, 605

Wardle, M. 1991, MNRAS, 251, 119

Zweibel, E. G. 2002, ApJ, 567, 962 


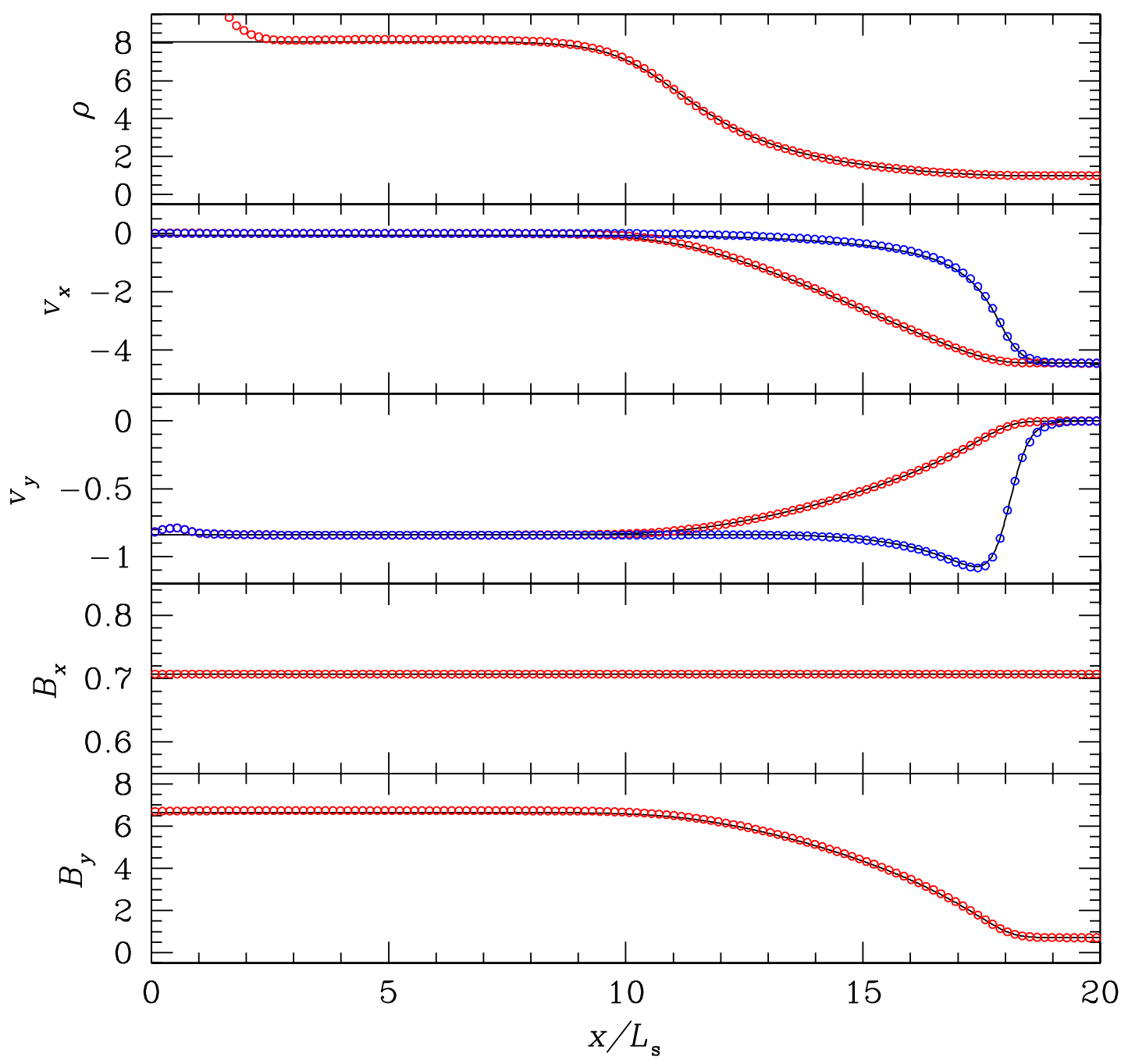

Fig. 1.- (a) Structure of an oblique C-type shock with $M=50, M_{\mathrm{A}}=5$ and $\theta=\pi / 4$. Profiles of neutral density, neutral (red) and ion (blue) velocity components, and magnetic field components from the numerical calculation are marked with open circles. The solid lines represent the analytic solution for the steady-state C-type shock. The calculation has been done in a square box with size $L=20 L_{s}$ using $128^{2}$ cells. (b) Same as in (a) except for $M=100, M_{\mathrm{A}}=10$, and $\theta=\pi / 4$. 


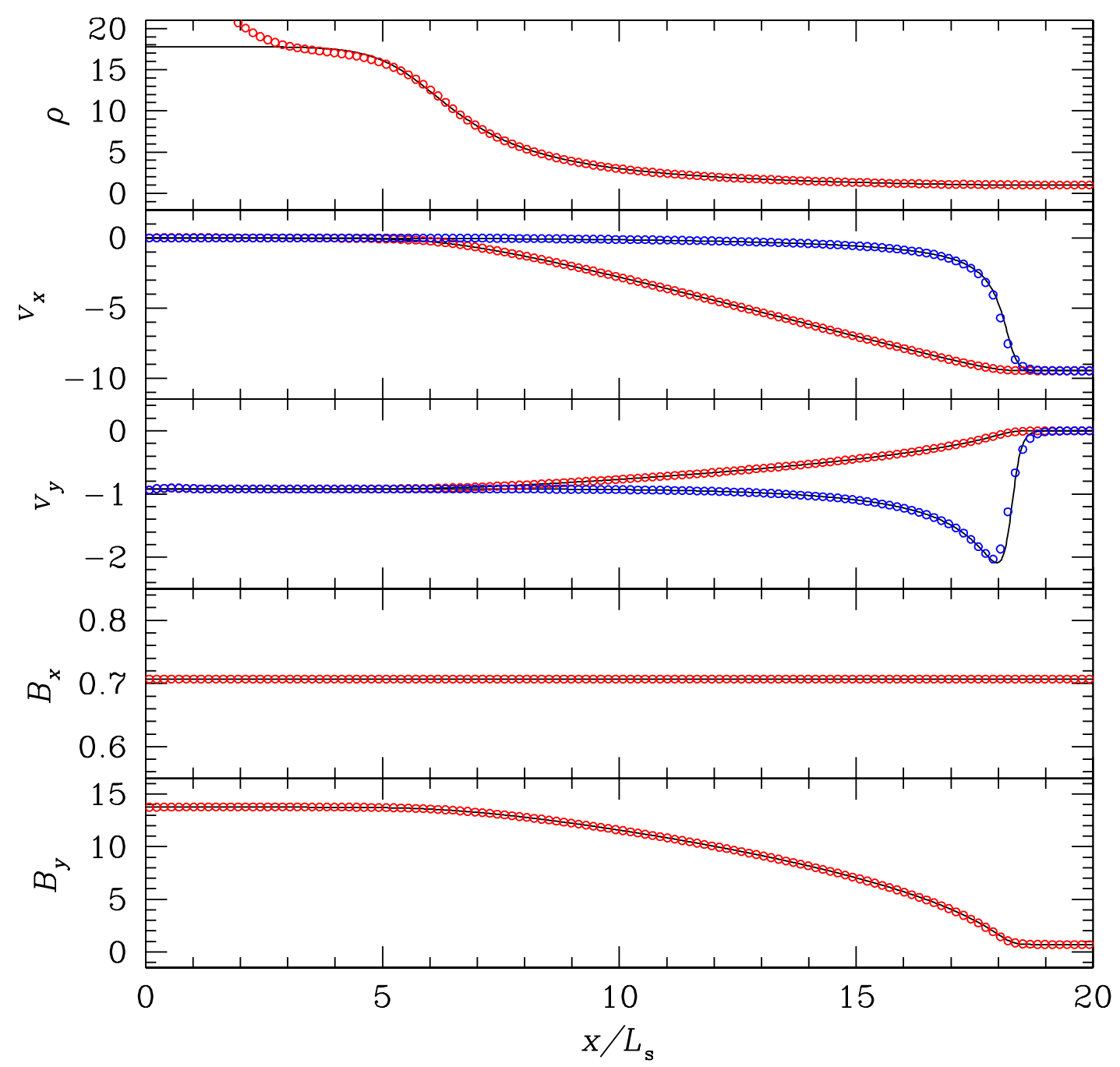



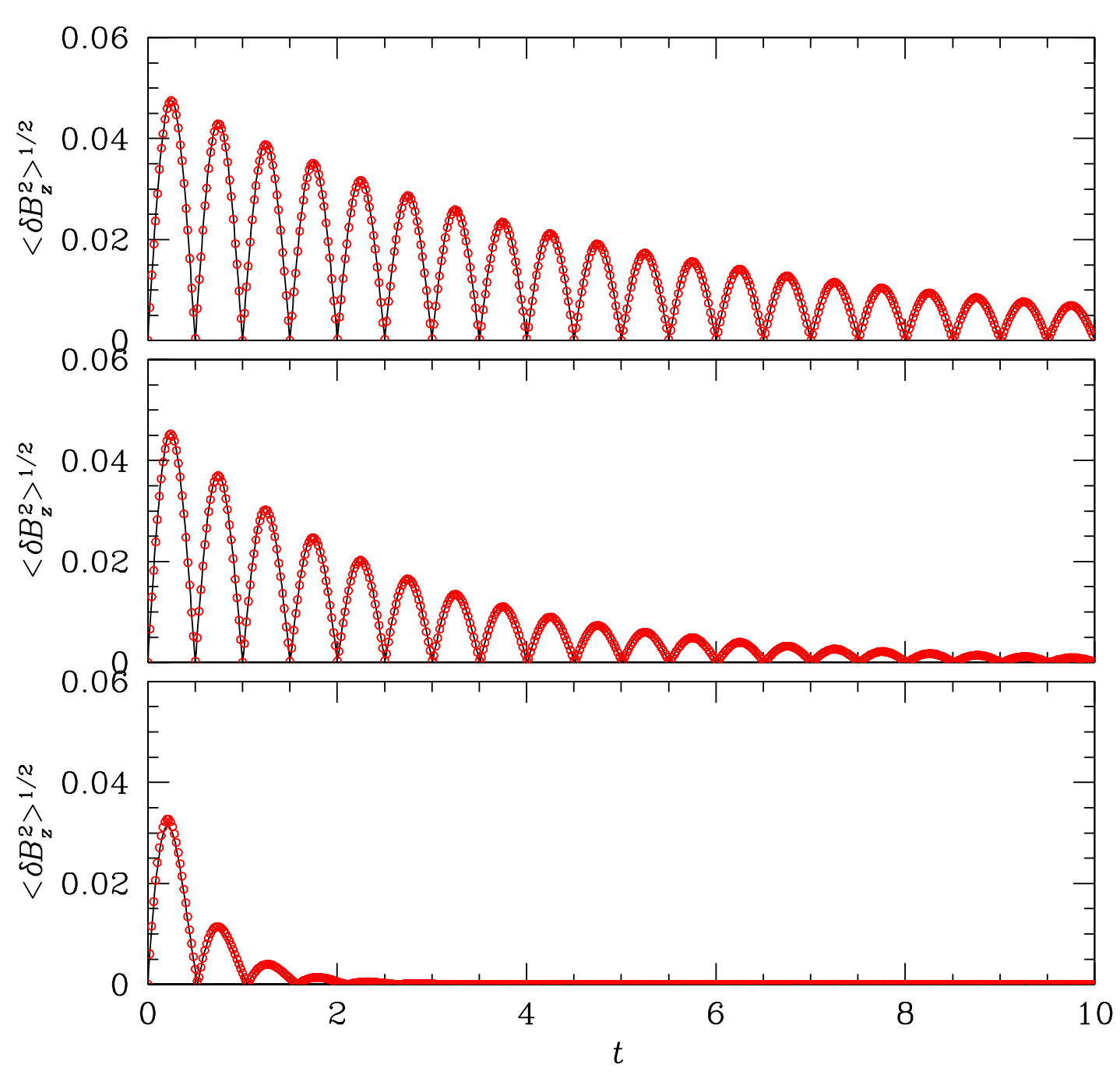

Fig. 2.- Time evolution of the spatially averaged root mean square magnetic field in the $z$ direction for $\gamma=1000$ (top), 500 (middle), and 100 (bottom) in the test of the decay of Alfvén waves. Our numerical results (open circles) are compared to the theoretical predictions (solid lines). The calculations have been done in a cube box with size $L=1$ using $128^{3}$ cells. Time is expressed in units of the sound wave crossing time, $L / a$. 


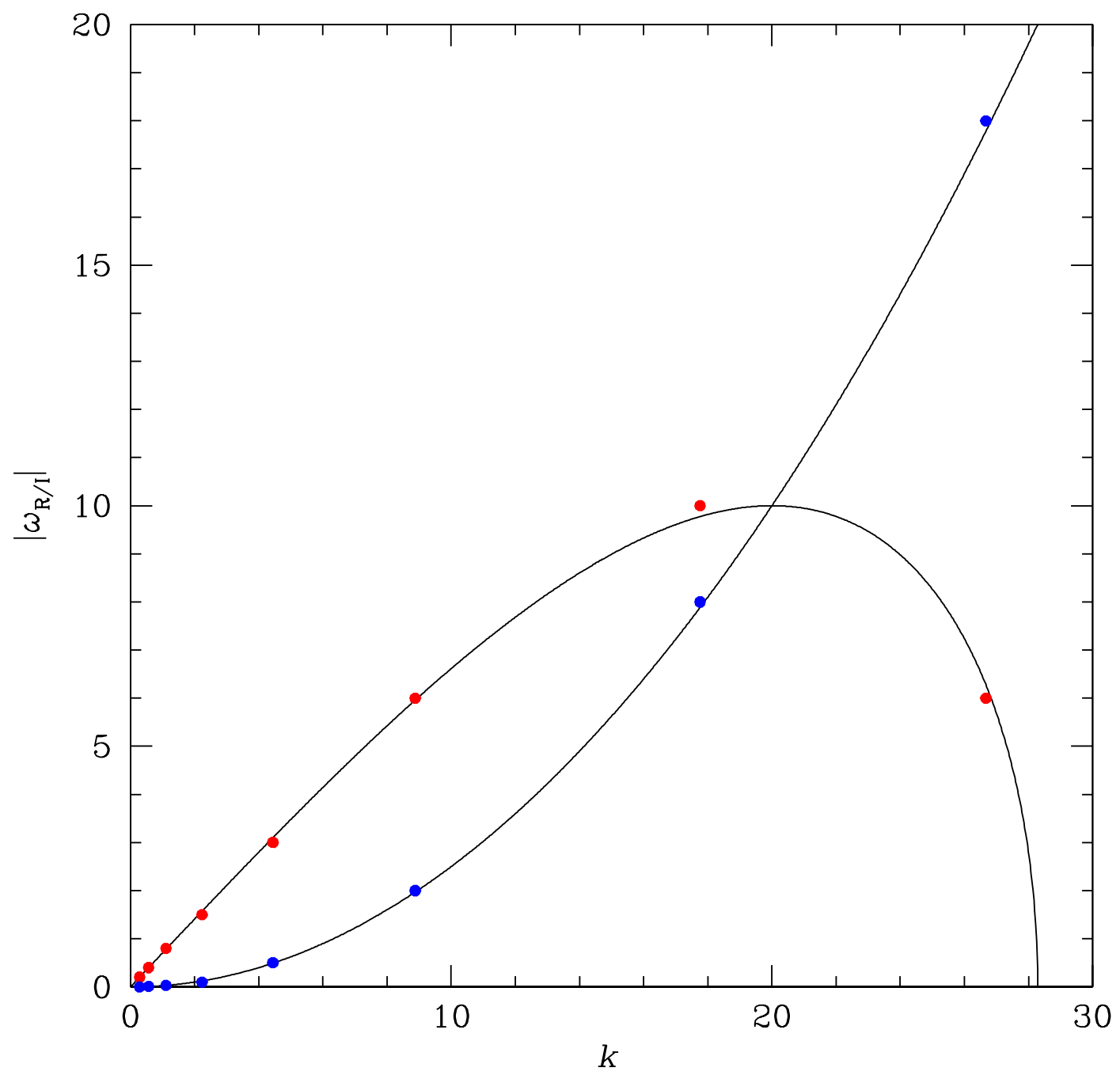

Fig. 3.- Oscillation frequencies $\omega_{R}$ and decay rates $\omega_{I}$ collected from the oscillation of standing waves with different wavenumbers for $\gamma=100$ in the test of the decay of Alfvén waves. Oscillation frequencies (red) and decay rates (blue) are marked with filled circles and the analytic solutions are drawn as solid lines. The calculations were done in a cubic box of size $L=1$ using $128^{3}$ cells. 

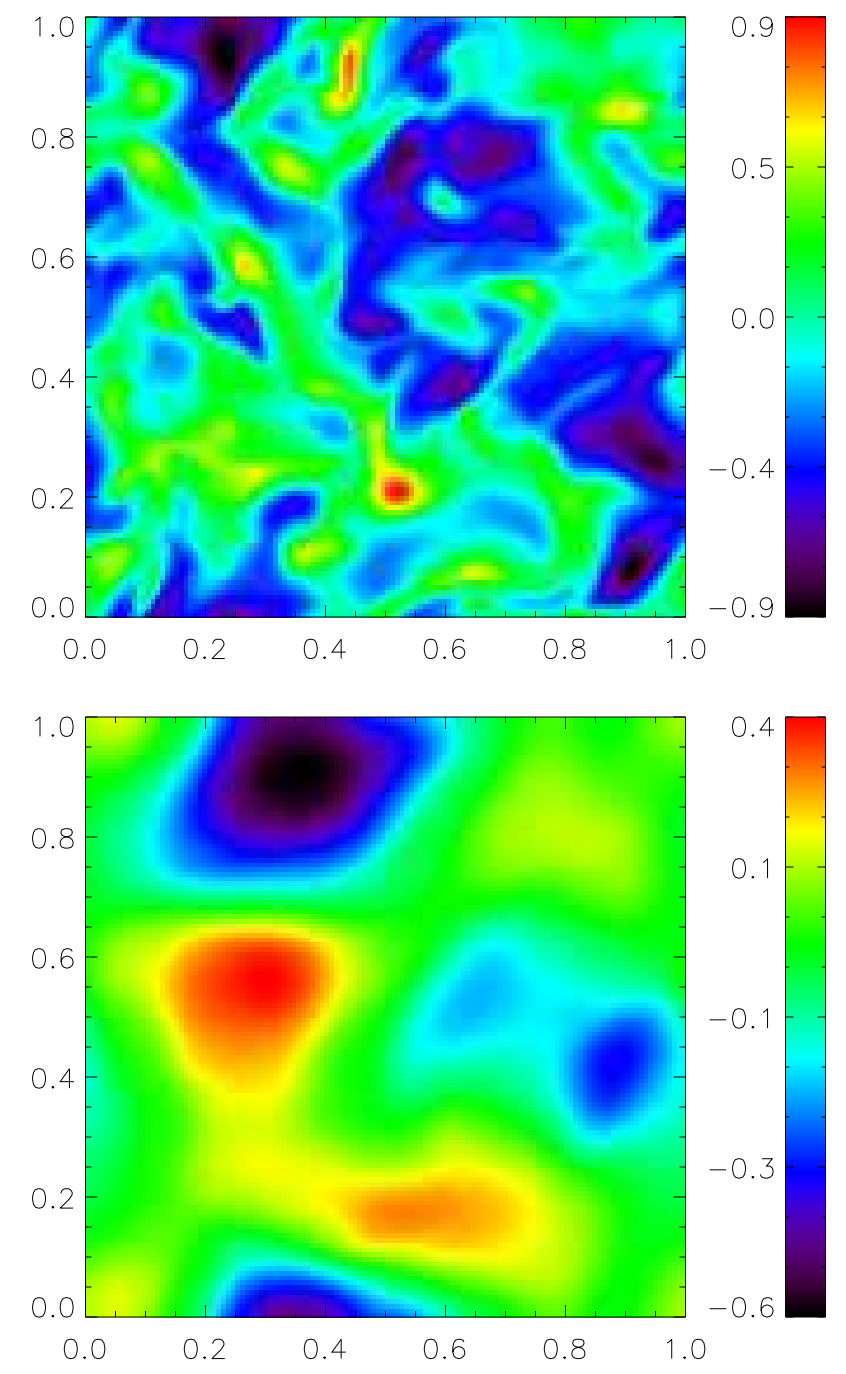

Fig. 4.- (a) Images of the neutral density for models D1 (top) and D3 (bottom) at $t=1 t_{f}$ in the MHD turbulence simulations. The simulations have been done in a cubic, periodic box with size $L=1$ using $128^{3}$ cells, and the images shown are slices through the $x-y$ plane at $z=0.5$. The color bars are drawn in logarithmic (base 10) scales. (b) Same as in (a) except for models F1 (top) and F3 (bottom) at $t=1 t_{f}$. 

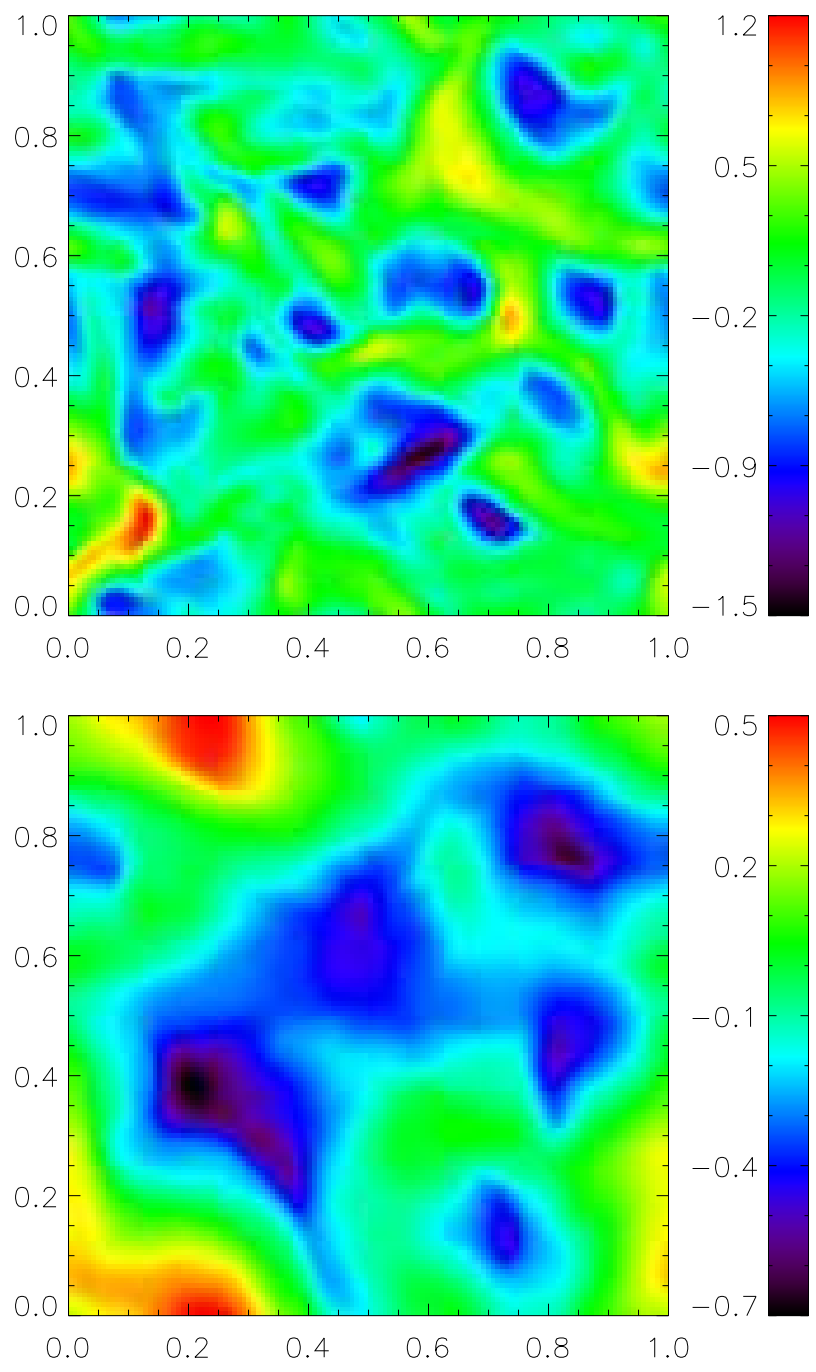


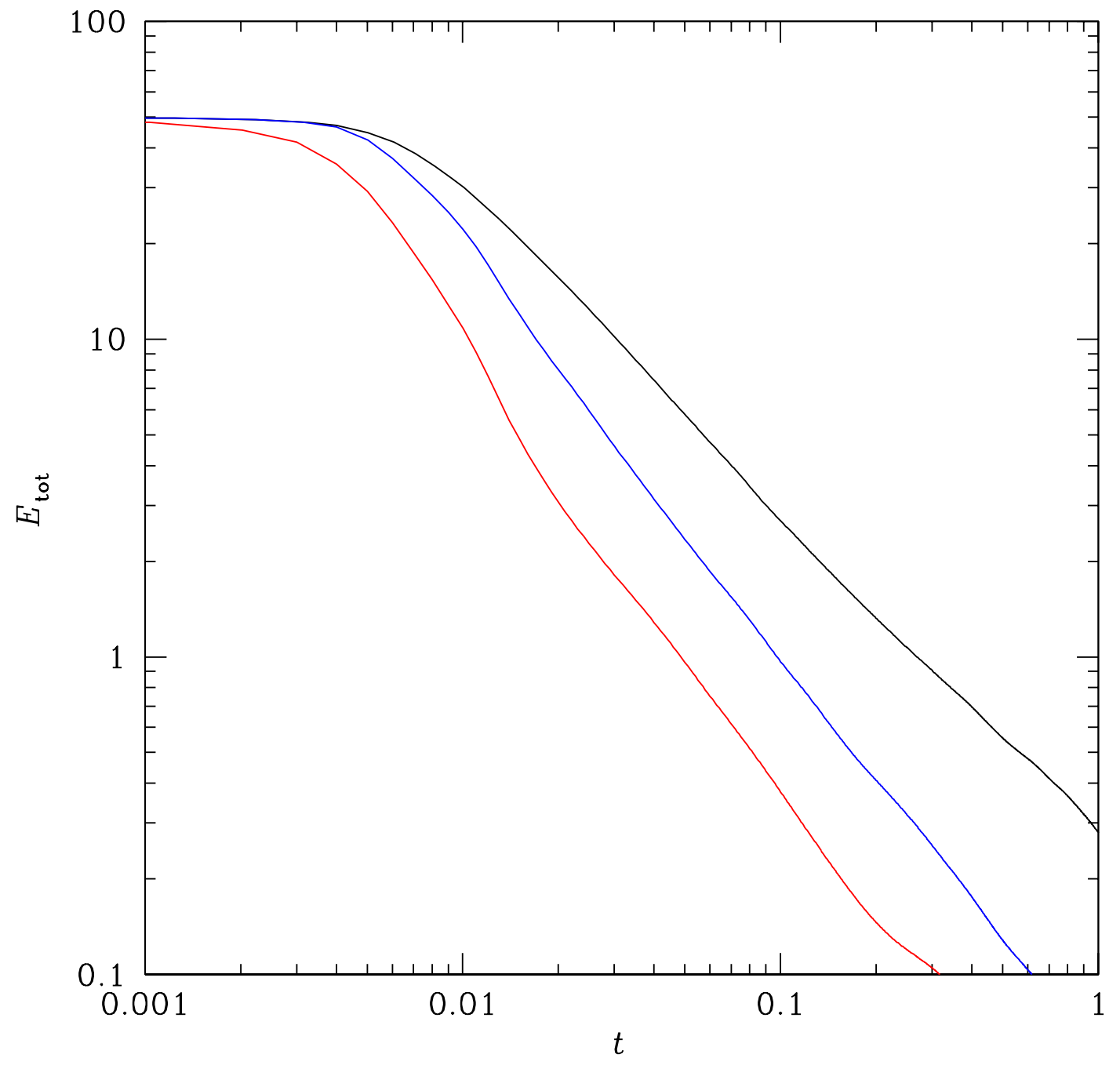

Fig. 5.- (a) Time evolution of the total energy for the decaying models D1 (black), D2 (blue), and D3 (red) in the MHD turbulence simulations. The simulations have been done in a cubic, periodic box with size $L=1$ using $128^{3}$ cells. Time is expressed in units of the sound wave crossing time, $L / a$. (b) Same as in (a) except for the forced models F1 (black), F2 (blue), and F3 (red). 


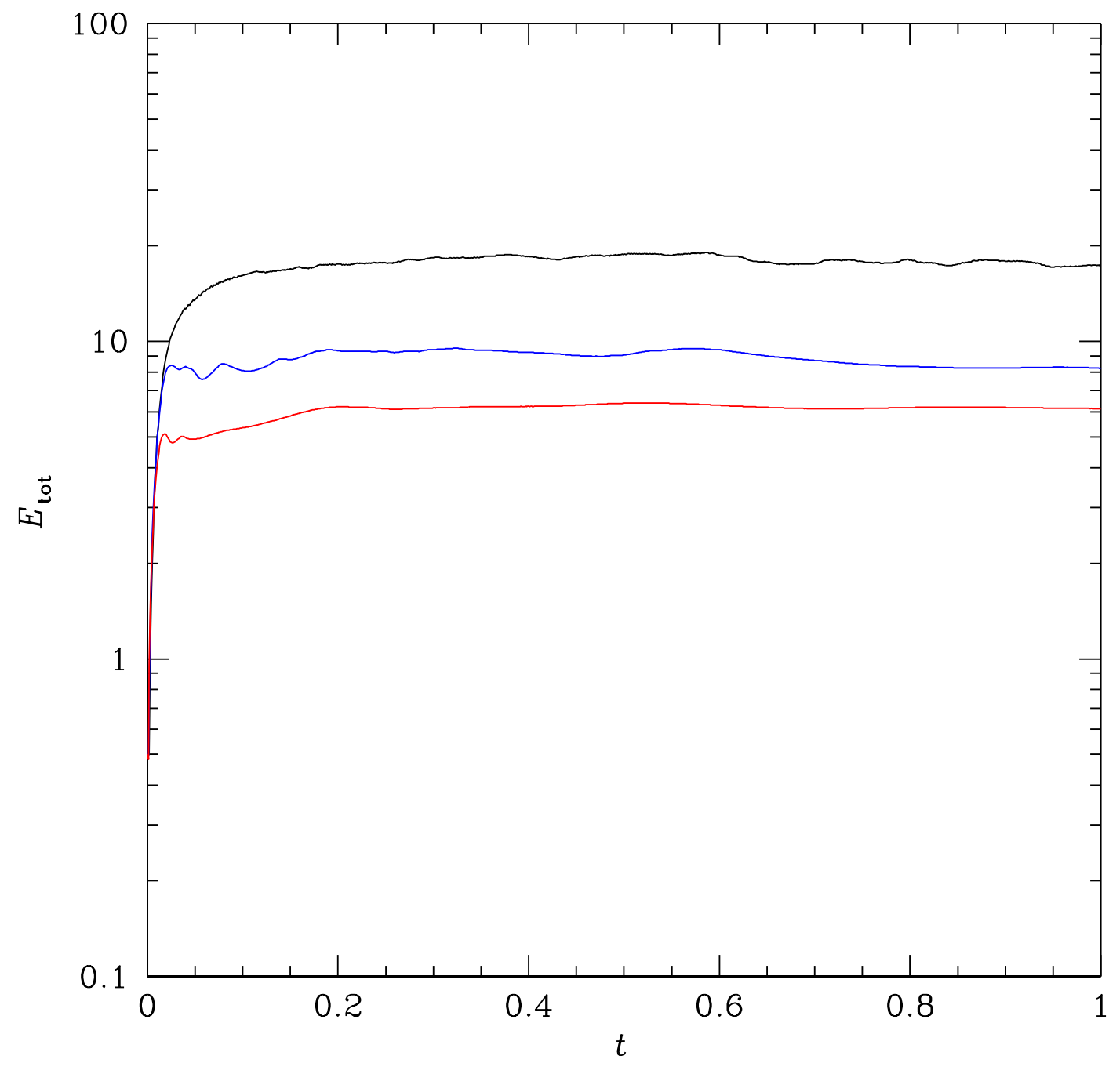


Table 1. Parameters for MHD Turbulence Simulations

\begin{tabular}{ccccccccc}
\hline \hline Model & $\beta$ & $\gamma$ & $M_{\mathrm{A}}$ & $\tau$ & $R_{\mathrm{AD}}$ & $l_{\mathrm{AD}}$ & $t_{f}$ & $t_{\text {end }}$ \\
\hline D1(F1) & 1 & $\infty$ & $10(1)$ & 0 & $\infty$ & 0 & $0.1(1)$ & $10(1) t_{f}$ \\
D2(F2) & 1 & 1000 & $10(1)$ & 0.1 & $100(10)$ & $0.01(0.1)$ & $0.1(1)$ & $10(1) t_{f}$ \\
D3(F3) & 1 & 100 & $10(1)$ & 1 & $10(1)$ & $0.1(1)$ & $0.1(1)$ & $10(1) t_{f}$ \\
\hline
\end{tabular}

Note. - Here models D1 to D3 represent decaying turbulence and models F1 to F3 denote forced turbulence. All the decaying and forced models have been done in pairs, and the different values for both models are distinguished using parentheses. 